\title{
Modelado, simulación y control de un reactor en la producción de cloruro de aluminio
}

\section{Modeling, simulation and control of a reactor for the production of aluminum chloride}

\author{
Argemiro Palencia Diaz'1, Javier Andrés Carpintero Durango², Jonathan Fabregas Villegas ${ }^{3}$ \\ ${ }^{1}$ Magíster en Ingeniería Mecánica, Profesor de Tiempo Completo, grupo GIIMA, \\ Universidad Autónoma del Caribe, apalencia@uac.edu.co \\ 2,3 Ingeniero Mecánico, Profesor Catedrático, Universidad Autónoma del Caribe, grupo GIIMA
}

Recibido 03/05/12, Aceptado 18/11/2012

\section{RESUMEN}

El Cloruro de Aluminio es una sustancia importante como medio de transferencia de calor y como fluido de trabajo en ciclos termodinámicos. Esta investigación consiste en la implementación de una estrategia de control clásico sobre un proceso de producción de Cloruro de Aluminio a partir de materias primas recicladas. Las ecuaciones diferenciales desarrolladas con base a los fenómenos fisicoquímicos que ocurren dentro del volumen de control fueron suficientes para representar la dinámica del sistema y por tanto deducir la velocidad de respuesta y la sensibilidad de este proceso.

Como indicador de la eficiencia del controlador evaluado para la simulación, se utilizó la integral absoluta del error (IAE) teniendo en cuenta la dinámica del proceso. Los resultados de esta investigación demuestran que la estrategia de control tiene buen desempeño para todas las situaciones estudiadas.

Palabras clave: Control clásico, IAE, Cloruro de aluminio.

\begin{abstract}
Aluminum Chloride is an important substance as heat transfer medium and as working fluid for thermodynamic cycles. This work involves the implementation of a classical control strategy on a production process of aluminum chloride from recycled raw materials. The differential equations developed based with the physicochemical phenomena that occur within the control volume were sufficient to represent the system dynamics and therefore deduce the speed of response and sensitivity of this process.
\end{abstract}

As an indicator of the efficiency of the controller, we used the integral absolute error (IAE) taking into account the dynamics of the process. The results of this investigation show that the control strategy has good performance for all cases studied.

Keywords: Classic control, IAE, Aluminum chloride.

\section{INTRODUCCIÓN}

Para iniciar este proceso se deposita una cantidad estequiometricamente efectiva de chatarra de aluminio dentro de un reactor CSTR, para que el paso de forma continua de ácido clorhídrico al 22\% grado industrial genere el denominado tricloruro de aluminio. Como subproducto, la reacción libera gas hidrogeno a los alrededores por tratarse de una reacción óxido-reducción de desplazamiento de hidrogeno. Dado que para establecer de forma clásica la estrategia de control PID, se necesita deducir un modelo dinámico del proceso, se hizo uso de los resultados de B.I. ITA, M. NSi y U.S Simon [1] para interpretar la cinética de la reacción. 
Posteriormente, las ecuaciones diferenciales deducidas de este modelo dinámico fueron solucionadas en estado estable a fin de conocer la magnitud de las variables que describen los fenómenos fisicoquímicos inherentes al sistema. Por último, se acude a la herramienta MATLAB® para evaluar la calidad del modelo y el rendimiento de la estrategia de control clásica utilizando perturbaciones y cambios del punto de control.

Son varias las investigaciones relacionadas con las características del cloruro de aluminio así como del control de su proceso de producción y su utilización en múltiples aplicaciones, es así como Narendra V. et al [2] realizaron análisis con el cloruro de aluminio cuando se utiliza en bio-prótesis para mejorar las propiedades de la misma alcanzándose buenos resultados. Por otro lado, Yu H. et al [3] observaron el comportamiento de un reactor para granulación cuando se incluía cloruro de aluminio en el proceso, logrando reducciones en el tiempo de permanencia en el reactor de hasta $30 \%$.

Gabelich Ch. et al [4] analizaron la influencia de distintos componentes entre los que se encuentra el cloruro de aluminio en la osmosis inversa, obteniendo resultados adecuados para las características requeridas. El cloruro de aluminio se ha utilizado en muchas aplicaciones, aparecen estudios clínicos sobre su influencia en cartílagos y huesos como los reportados por Denisov A. [5] y Xin L. et al [6], los cuales logran un buen desempeño para los casos estudiados.

Ghafari S. et al [7] estudiaron el comportamiento del cloruro de aluminio en el tratamiento de estabilización de sustancias complejas, los resultados se comparan con los logrados con la utilización de un compuesto convencional. Li F. et al [8] presentaron un nuevo método para la producción de cloruro de aluminio con mayores niveles de pureza, en sus resultados muestran que el cloruro desarrollado es más eficiente para la remoción de la turbiedad que el componente convencional. Meiying L. y Yongbo S. [9] muestran los resultados de su experimentación en un reactor para el proceso de coagulación, teniéndose al final de sus pruebas que el proceso utilizando cloruro de aluminio es más económico y que cumple con los estándares de calidad requeridos. Zhang Z. et al [10] desarrollaron un estudio experimental para la producción de cloruro de aluminio a escala industrial, en sus resultados muestran que su producto es mejor en general que los productos convencionales. Liu $\mathrm{H}$. et al [11] realizaron una investigación experimental en la cual comparan los resultados alcanzados en el proceso de tratamiento de agua del cloruro de aluminio y otras alternativas, reportan las concentraciones adecuadas de cada componente para lograr alcanzar los estándares deseados en términos de turbiedad y remoción de impurezas.
Como se puede apreciar, existen varias líneas de investigación en el campo del cloruro de aluminio que van desde su producción hasta aplicaciones medicas y su uso para procesos de tratamiento de residuos. Las siguientes secciones muestran un estudio computacional del comportamiento de una estrategia de control clásico en la producción de cloruro de aluminio.

\section{METODOLOGÍA}

\subsection{Modelado dinámico del sistema}

\subsubsection{Descripción del proceso}

Se tiene un flujo volumétrico de ácido clorhídrico al 22\% sobre un tanque que contiene $300 \mathrm{~kg}$ de chatarra de aluminio. Las principales perturbaciones del sistema son la temperatura y la concentración de la corriente de ácido clorhídrico que entra al reactor. Se asume que las propiedades termo físicas del fluido se mantienen constantes, así como el volumen de reacción en el reactor. La variable sobre la que se va a ejecutar la acción de control es la temperatura de salida del reactor y la variable manipulada para las estrategias de control es el flujo volumétrico de ácido clorhídrico. La figura 1 presenta el esquema del sistema productor del fluido de trabajo.

Figura 1. Reactor CSTR

Figure 1. CSTR reactor

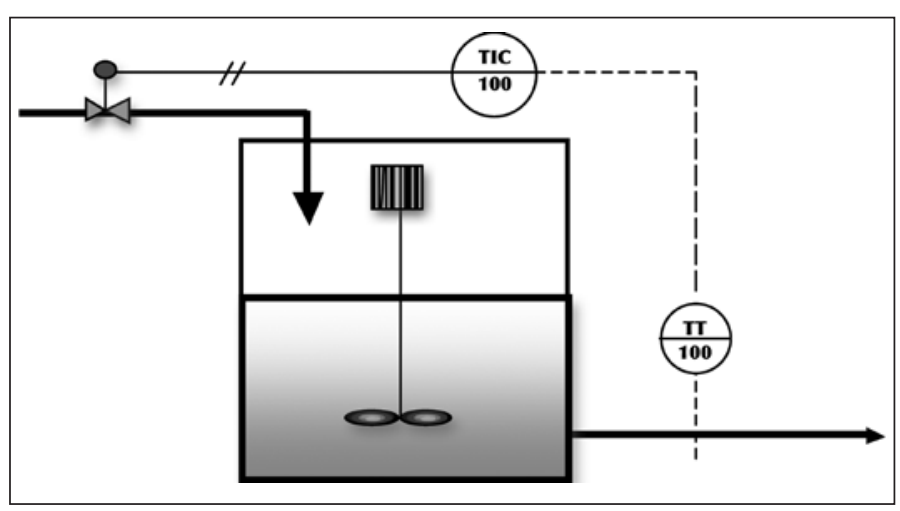

El proceso se da de la siguiente manera de acuerdo al concepto de la energía libre de Gibbs y a la ecuación iónica descrita:

$$
\begin{gathered}
\mathrm{AlCl}_{3}(\mathrm{~s}) \stackrel{\mathrm{H}_{3} \mathrm{O}}{\rightarrow} \mathrm{Al}^{3+}(\mathrm{ac})+3 \mathrm{Cl}^{-}(\mathrm{ac}) \\
\Delta \mathrm{G}=\Delta H_{r x n}-\mathrm{T} \Delta S \\
\Delta \mathrm{G}=(-1049.4 \mathrm{k})-((303.15 \mathrm{k}) \times(-290.36 \mathrm{j} / \mathrm{k})) \\
\Delta \mathrm{G}=(-1049.4 \mathrm{kj})+88.02 \mathrm{kj} \\
\Delta \mathrm{G}=-961.37 \mathrm{kj}
\end{gathered}
$$


Esto garantiza que la reacción procederá de forma espontánea al contacto de los reactivos.

\subsubsection{Modelo Dinámico del Sistema}

Para el desarrollo del siguiente modelo matemático se realizaron las siguientes consideraciones:

- $\quad$ La reacción es irreversible, con la siguiente estequiometria:

$\mathrm{s}+6 \mathrm{HClac} \Delta 2 \mathrm{AlCl} 3 \mathrm{ac}+3 \mathrm{H} 2 \mathrm{ac}(2)$

- $\quad$ El proceso es adiabático.

- $\quad$ La reacción ocurre a volumen, densidad y calor específico constante.

Se presenta el modelo dinámico del sistema de la siguiente manera:

Balance molar de componente clave ácido clorhídrico:

La hidrólisis del agua por la presencia del ión aluminio es despreciable.

$\mathrm{C}_{\mathrm{HCl}}(\mathrm{t}) \mathrm{f}_{\mathrm{HCl}}(\mathrm{t})-\mathrm{C}_{\text {out HCl}}(\mathrm{t}) \mathrm{F}-\mathrm{V}_{\mathrm{rxn}} \mathrm{R}_{\mathrm{HCl}}(\mathrm{t})=\mathrm{V} \frac{\mathrm{d}}{\mathrm{dt}}$ [Cout $\left._{\mathrm{HVCl}}(\mathrm{t})\right]$

1ec, 2inc) $\mathrm{R}_{\mathrm{HCl}}(\mathrm{t}), \mathrm{C}_{\text {out } \mathrm{HCL}}(\mathrm{t})$

Dónde:

$\mathrm{C}_{\mathrm{HCl}}(\mathrm{t})$ : concentración inicial de ácido clorhídrico

$\mathrm{f}_{\mathrm{HCl}}(\mathrm{t})$ : flujo de entrada de ácido clorhídrico

$\mathrm{C}_{\text {out } \mathrm{HCl}}(\mathrm{t})$ : concentración final de ácido clorhídrico

$\mathrm{f}: \quad$ flujo a la salida del reactor

$\mathrm{V}_{\mathrm{rxn}}: \quad$ volumen de la reacción

$\mathrm{R}_{\mathrm{HCl}}(\mathrm{t})$ : tasa de reacción del proceso

Tasa de reacción del proceso:

$$
\mathrm{R}_{\mathrm{HCl}}(\mathrm{t})=\mathrm{A}_{\mathrm{o}} \mathrm{C}_{\text {ountHCL }}(\mathrm{t}) \mathrm{e}^{\frac{-\mathrm{E}}{\mathrm{RT}(\mathrm{t})}}(2 \mathrm{ec}, \text { einc) } \mathrm{T}(\mathrm{t})
$$

Dónde:

Ao: constante de frecuencia

$\mathrm{R}: \quad$ constante de los gases ideales

$\mathrm{T}(\mathrm{t})$ : temperatura de la reacción

E: energía de activación

Balance de energías del proceso:

$$
\rho f_{\mathrm{HCl}}(\mathrm{t}) \mathrm{Cp} \mathrm{p}_{\mathrm{HCl}} \mathrm{T}(\mathrm{t})-\rho \mathrm{CpfT}(\mathrm{t})-\mathrm{V} \Delta_{\mathrm{rxn}} \mathrm{R}_{\mathrm{HCl}}(\mathrm{t})=\mathrm{V} \rho \mathrm{Cv} \frac{\mathrm{d}}{\mathrm{dt}}[\mathrm{T}(\mathrm{t})]
$$

(3ec, 3inc) OK

Donde:
Las ecuaciones 3 a 5 son suficientes para describir la dinámica del proceso. La tabla 1 presenta los valores en estado estable del proceso.

Tabla 1. Condición en estado estacionario del proceso Table 1. Steady state condition

\begin{tabular}{|c|c|c|}
\hline SíMBOLO & UNIDAD & MAGNITUD \\
\hline $\mathrm{f}_{\mathrm{HCl}}$ & $\mathrm{m}^{3} / \mathrm{s}$ & $4,10 \mathrm{E}-03$ \\
\hline $\mathrm{C}_{\mathrm{HCl}}$ & $\mathrm{mol} / \mathrm{m}^{3}$ & 1184 \\
\hline$\rho_{\mathrm{HCl}}$ & $\mathrm{kg} / \mathrm{m}^{3}$ & 1024,68896 \\
\hline $\mathrm{T}_{\mathrm{HCl}}$ & $\mathrm{K}$ & 303,15 \\
\hline $\mathrm{Cp}_{\mathrm{HCl}}$ & $\mathrm{kJ} / \mathrm{kg} \cdot \mathrm{K}$ & 3,84672458 \\
\hline $\mathrm{A}_{0}$ & $\mathrm{seg}^{-1}$ & 291 \\
\hline $\mathrm{Ea}$ & $\mathrm{J} / \mathrm{mol}$ & 33000 \\
\hline $\mathrm{R}$ & $\mathrm{J} / \mathrm{K} \cdot \mathrm{mol}$ & 8,134 \\
\hline$\Delta \mathrm{Hrxn}$ & $\mathrm{kJ} / \mathrm{mol}_{\mathrm{HCl}}$ & $-349,6$ \\
\hline $\mathrm{f}$ & $\mathrm{m} / \mathrm{s}$ & 0,00319528 \\
\hline$\rho$ & $\mathrm{kg} / \mathrm{m}^{3}$ & 1314,5983 \\
\hline $\mathrm{Cp}$. & $\mathrm{kJ} / \mathrm{kg} \cdot \mathrm{K}$ & 3,7 \\
\hline $\mathrm{Vrxn}$ & $\mathrm{m}^{3}$ & 0.65 \\
\hline $\mathrm{To}$ & $\mathrm{K}$ & 372,039763 \\
\hline $\mathrm{C}_{\mathrm{HClo}}$ & $\mathrm{mol} / \mathrm{m}^{3}$ & 727,76946 \\
\hline $\mathrm{R}_{\mathrm{HCl}}$ & $\mathrm{mol} / \mathrm{s}$ & 3,89011833 \\
\hline
\end{tabular}

El software MATLAB® permitió la validación del modelo anteriormente presentado. La figura 2 se observa el comportamiento de la concentración de ácido clorhídrico a la salida del reactor ante incrementos de $+5 \%$ de la temperatura de la corriente de entrada de ácido. Dado que la reacción es exotérmica, el principio de Le chatelier establece que los procesos de esta naturaleza se ven favorecidos por disminución de la temperatura de los alrededores. Tal como se esperaba, la concentración aumenta debido al aumento de la temperatura dentro de la corriente de entrada.

Figura 2. Comportamiento del sistema para cambio de temperatura

Figure 2. System behavior to temperature change

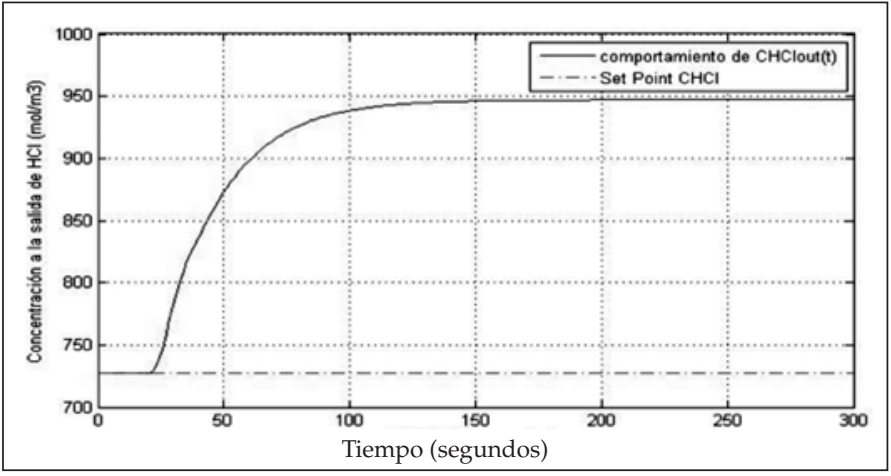


La figura 3 presenta el comportamiento de la temperatura del proceso frente a perturbaciones en las variables de entrada. Se aprecia que la temperatura de salida del reactor muestra mayor sensibilidad ante cambios en la temperatura de entrada que la concentración de entrada de ácido clorhídrico.

Figura 3. Respuesta de la temperatura de salida del reactor ante perturbaciones en las entradas del proceso

Figure 3. Behavior of the reactor temperature to disturbances in the process

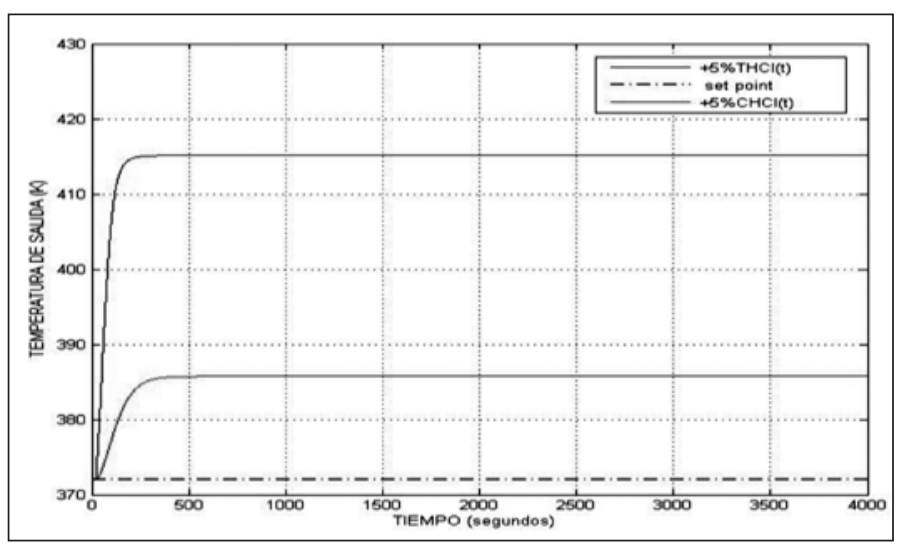

\subsection{Sintonización del proceso}

Para la sintonización del proceso, se hizo una prueba de caracterización del sistema a controlar por medio de la variación del flujo de la corriente de ácido desde su estado estable hasta un incremento del $20 \%$. Tal acción permitió ajustar el modelo dinámico a uno de primer orden más tiempo muerto por el método FIT 3 (tabla 2).

Tabla 2. Sintonía del controlador

Table 2. Controller tuning

\begin{tabular}{|c|c|c|c|}
\hline \multicolumn{2}{|c|}{$\begin{array}{c}\text { Parámetros } \\
\text { del proceso }\end{array}$} & \multicolumn{2}{c|}{$\begin{array}{c}\text { Ajuste de integral mínima de error } \\
\text { para perturbaciones }\end{array}$} \\
\hline $\mathrm{K}$ & $-0,409$ & $\mathrm{KC}$ & $-19,3407844$ \\
\hline$\tau$ & 49,3829 & $\tau_{\mathrm{I}}$ & 14,0220213 \\
\hline $\mathrm{t} 0$ & 7,72931 & $\tau_{\mathrm{D}}$ & 2,88963501 \\
\hline
\end{tabular}

\section{RESULTADOS}

La figura 4 presenta el comportamiento de la estrategia PID para una perturbación de $+5 \%$. El controlador actúa sobre La temperatura de salida con una mayor oscilación cuando se perturba la temperatura de entrada, que cuando se modifica la concentración de ácido. En la figura 5 se observa que para cambios en el Set Point, el controlador responde con menor overshoot que a diferencia del control para perturbaciones en la entrada del proceso.

La tabla 3 presenta los resultados de la evaluación del controlador PID por medio del indicador de la Integral Absoluta del Error o IAE, donde se observa que por la naturaleza de la perturbación, el controlador posee mejor comportamiento ante cambios en la concentración de ácido clorhídrico que para cambios de temperatura cuando el incremento es del $+5 \%$ de su valor inicial.

Figura 4. Comportamiento de controlador PID frente a un cambio de $+5 \%$

Figure 4. Behavior of the PID controller to a change of $+5 \%$

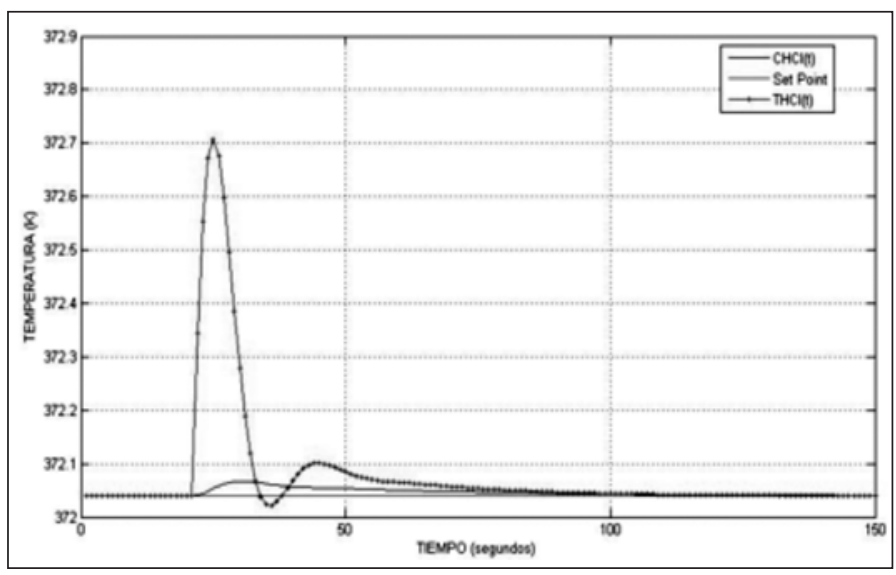

Figura 5. Comportamiento de controlador PID frente a un cambio de $+5 \%$ en el Set Point

Figure 5. PID controller behavior to a change of $+5 \%$ in the Set Point

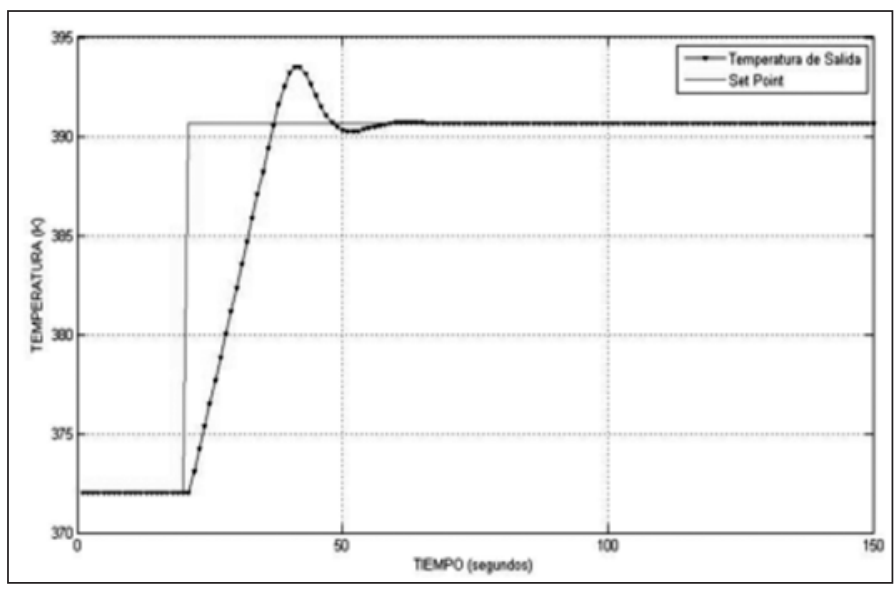


Tabla 3. Rendimiento de controlador PID

Table 3. PID controller performance

\begin{tabular}{|l|c|}
\hline \multicolumn{1}{|c|}{$\begin{array}{c}\text { Variables de entradas el reactor productor } \\
\text { de cloruro de aluminio }\end{array}$} & IAE \\
\hline Concentración de $\mathrm{HCl}$ en $5 \%$ perturbado & 2.247 \\
\hline $\begin{array}{l}\text { Temperatura de entrada de corriente para un } 5 \% \\
\text { en perturbación }\end{array}$ & 13.45 \\
\hline Cambio en el Set Point & 376.5 \\
\hline
\end{tabular}

Posteriormente, se siguió perturbando el proceso para observar la capacidad de control de controlador PID ante perturbaciones y cambios en el Set Point del sistema. La figura 6 presenta los resultados para perturbaciones en $-5 \%$ del valor inicial. Nótese que el proceso de control se comporta de forma inversa al que presenta la figura 4 .

Figura 6. Comportamiento ante un cambio en $-5 \%$ Figure 6. Behaviour to a change of $-5 \%$

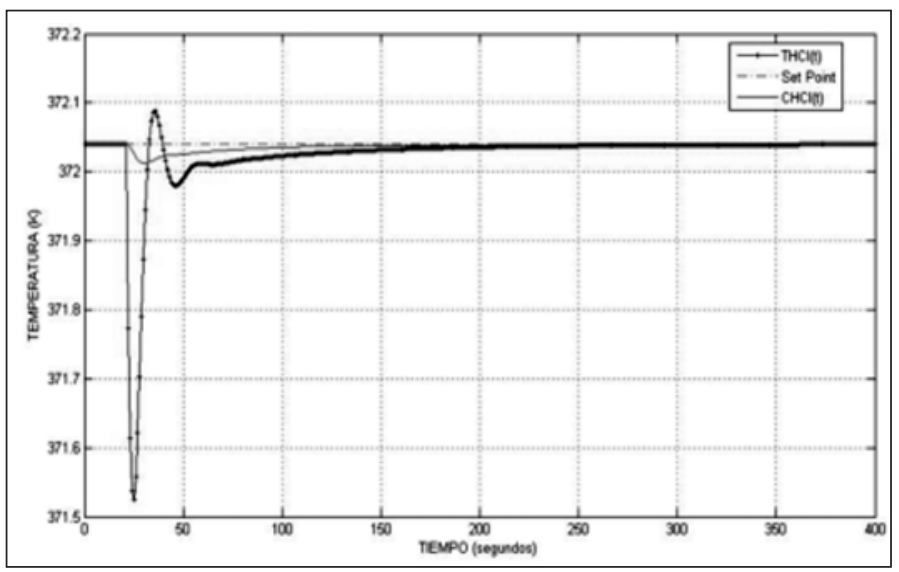

La figura 7 presenta el comportamiento del controlador PID ante cambios en el Set Point inferiores a $-5 \%$ de su valor inicial. Del mismo modo, la respuesta del controlador es contraria a la que presentó en la figura 5.

La tabla 4 presenta los resultados de la evaluación de la estrategia PID por medio del indicador de la Integral del Error Absoluto, IAE.

Se aprecia que la estrategia de control tiene mejor eficiencia para perturbaciones que posean incrementos porcentuales de su valor inicial.
Tabla 4. Evaluación de controlador en $-5 \%$

Table 4. Evaluation of the controller to $-5 \%$

\begin{tabular}{|l|c|}
\hline \multicolumn{1}{|c|}{$\begin{array}{c}\text { Variables de Entradas el reactor productor de } \\
\text { cloruro de aluminio }\end{array}$} & IAE \\
\hline Concentración de $\mathrm{HCl}$ en 5\% perturbado & 2.482 \\
\hline $\begin{array}{l}\text { Temperatura de entrada de corriente para un 5\% } \\
\text { en perturbación }\end{array}$ & 14.5 \\
\hline Cambio en el Set Point & 149.1 \\
\hline
\end{tabular}

Figura 7. Comportamiento del controlador PID ante cambios en el Set Point en $-5 \%$

Figure 7. PID controller behavior to changes in the set point to $-5 \%$

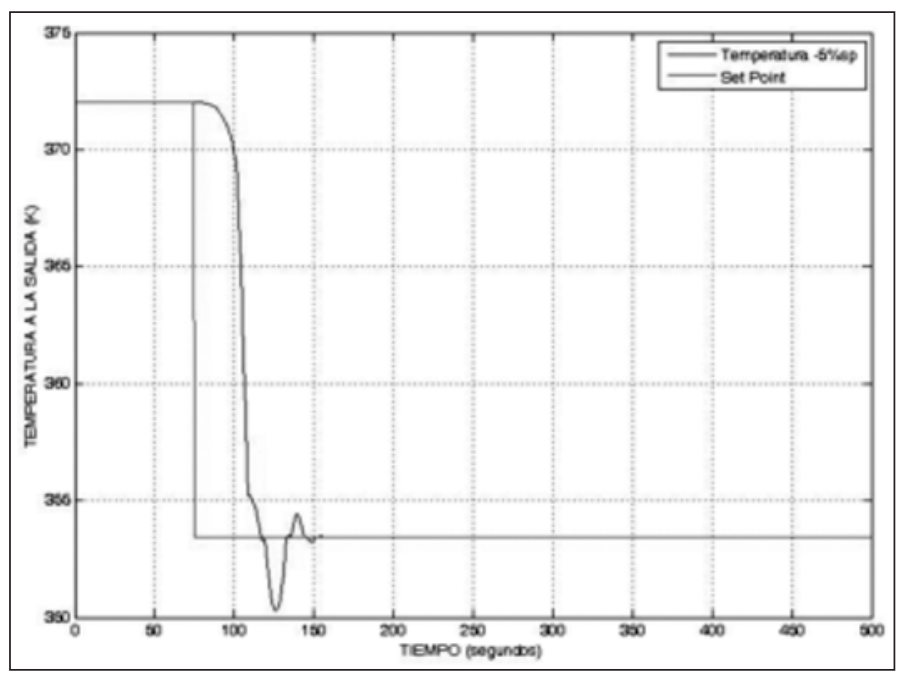

Se estudió el comportamiento del proceso para un incremento tanto en perturbaciones como para cambios del Set Point en $+10 \%$.Se observa en la tabla 5 que la tendencia que presenta el controlador para mantener el proceso bajo sus condiciones de estado estacionario es a duplicar la integral absoluta del error del proceso cuando se controlan la perturbación de concentración de ácido clorhídrico del sistema.

Tabla 5. Evaluación de controlador para $+10 \%$

Table 5. Evaluation of the controller to $+10 \%$

\begin{tabular}{|l|c|}
\hline \multicolumn{1}{|c|}{$\begin{array}{c}\text { Variables de Entradas el reactor productor } \\
\text { de cloruro de aluminio }\end{array}$} & IAE \\
\hline Concentración de $\mathrm{HCl}$ en $10 \%$ perturbado & 4.27 \\
\hline $\begin{array}{l}\text { Temperatura de entrada de corriente para un } \\
10 \% \text { en perturbación }\end{array}$ & 20.02 \\
\hline Cambio en el Set Point & 1303 \\
\hline
\end{tabular}




\section{CONCLUSIONES}

- A lo largo del estudio se evalúa la capacidad de control que posee una estrategia PID frente a la presencia de perturbaciones y ante cambios del Set Point. En este proceso se necesitó la ayuda de herramientas computacionales matemáticas para simular la respuesta del modelo dinámico del reactor. Además, de la caracterización termo física de la reacción para determinar los valores en estado estacionario de la reacción estudiada.

- Después de los análisis se tiene que la temperatura de entrada de ácido clorhídrico influye en mayor medida que la concentración del mismo, al momento de producir cloruro de aluminio, porque la temperatura del reactor mostró mayor sensibilidad para esta variable que para la concentración de entrada de ácido. El proceso responde a la acción de equilibrios químicos establecido por Le chatelier para reacciones exotérmicas.

- En relación a las dos variables de entradas estudiadas, el proceso de control mostró mayor oscilación en su respuesta cuando se controlaban las perturbaciones hechas por la temperatura de entrada, que cuando se controlan las perturbaciones sobre la concentración de ácido clorhídrico. Las perturbaciones realizadas sobre la temperatura de entrada muestran menor capacidad de control por parte de la estrategia PID que a en el caso de los cambios en la concentración del ácido.

\section{REFERENCIAS}

[1] ITA, B. I., NSi M., and Simon, U.S., Corrosion inhibition of aluminum in hydrochloric acid by 2-acetylpyridine thiosemicarbazone derivatives, International Journal of Natural and Applied Sciences (IJNAS),1(1), 73-80.

[2] Narendra V., Matthew O., Frederick S. and Robert J., Mechanisms Of Elastin Calcification and its Prevention
With aluminum chloride pretreatment, Procedinlgs of The First Joint BMES/EMBS Conference, 1999, Atlanta.

[3] Yu, H., Fang, H., Tay, J., Enhanced Sludge Granulation in Upflow Anaerobic Sludge Blanket (UASB) Reactors by Aluminum Chloride, Chemosphere, 44, 31-36, 2001.

[4] Christopher, J., Kenneth, P. I., Fredrick, W., Ray, E., Minhaal, K., Suffetd, I., Control of residual aluminum from conventional treatment to improve reverse osmosis performance, Desalination, 190, 147-160, 2006.

[5] Denisov A., Morphological and Functional State of Major Salivary Glands under Conditions of Aluminum Chloride Excess in Drinking Water. Bulletin of Experimental Biology and Medicine, 148, 703-706, 2009.

[6] Wei, L., Li, Z., Chong, H., Qing, G., Yue S., Yan, L., Effects of Chronic Aluminum Exposure on the Collagen Metabolism of Bone and Cartilage in Rats, 978-1-4244-4713-8/10.

[7] Ghafari, S., Abdul H., Hasnain, M., Zinatizadehd, A., Application of response surface methodology (RSM) to optimize coagulation-flocculation treatment of leachate using poly-aluminum chloride (PAC) and alum, Journal of Hazardous Materials, 163, 650-656, 2009.

[8] Fengting, L., Jia-Qian, J., Shengju, W., Bingru, Z., Preparation and performance of a high purity poly-aluminum chloride, Chemical Engineering Journal, 156, 64-69, 2010.

[9] Meiying, L. and Yongbo, S., High Efficiency Flocculation Treatment of Gas Washing Wastewater from Iron and Steel Plant, 978-1-61284-459-6/11.

[10] Zhen, Z., Di, G., Liu, H., Li, X., Study on Industrial Synthesis of Poly Aluminum Chloride and Coagulation Effect with Different Basicities, 978-1-61284-340-7/11.

[11] Liu, H., Di, G., Zhen, Z., Coagulants Optimization of Low Turbidity Water Treatment in Feedwater Plant, 978-14244-5089-3/11. 